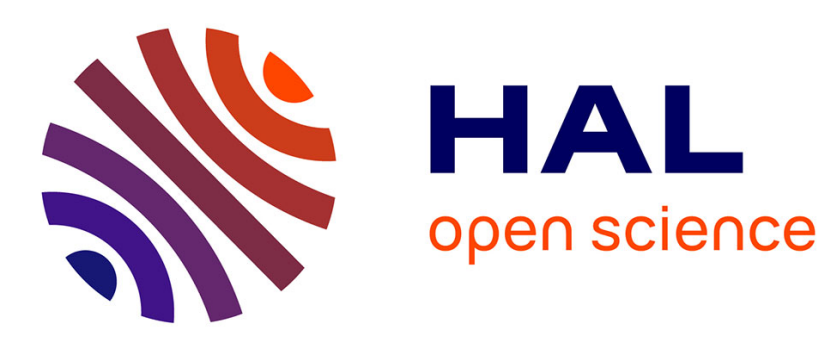

\title{
Interval-Passing Algorithm for Chemical Mixture Estimation
}

Ludovic Danjean, Bane Vasic, Michael Marcellin, David Declercq

\section{To cite this version:}

Ludovic Danjean, Bane Vasic, Michael Marcellin, David Declercq. Interval-Passing Algorithm for Chemical Mixture Estimation. IEEE Signal Processing Letters, 2013, 20 (9), pp.849-852. 10.1109/LSP.2013.2267656 . hal-00865690

\section{HAL Id: hal-00865690 https://hal.science/hal-00865690}

Submitted on 24 Sep 2013

HAL is a multi-disciplinary open access archive for the deposit and dissemination of scientific research documents, whether they are published or not. The documents may come from teaching and research institutions in France or abroad, or from public or private research centers.
L'archive ouverte pluridisciplinaire HAL, est destinée au dépôt et à la diffusion de documents scientifiques de niveau recherche, publiés ou non, émanant des établissements d'enseignement et de recherche français ou étrangers, des laboratoires publics ou privés. 


\title{
Interval-Passing Algorithm for Chemical Mixture Estimation
}

\author{
Ludovic Danjean*, Bane Vasić, Fellow, IEEE, \\ Michael W. Marcellin, Fellow, IEEE, and David Declercq, Senior Member, IEEE
}

\begin{abstract}
In this letter we propose a compressive sensing scheme for the mixture estimation problem in spectroscopy. We show that by applying an appropriate measurement matrix on the chemical mixture spectrum, we obtain an overall measurement matrix which is sparse. This enables the use of a low-complexity iterative reconstruction algorithm, called the interval-passing algorithm, to estimate the concentration of each chemical present in the mixture. Simulation results for the proportion of correct reconstructions show that chemical mixtures with a large number of chemicals present can be recovered.
\end{abstract}

Index Terms-Chemical mixture estimation, compressive sensing, iterative reconstruction algorithm.

\section{EDICS Category: DSP-SPARSE}

\section{INTRODUCTION}

$\mathbf{T}$ HE knowledge of the presence and/or concentration of a particular chemical in a solution or on a surface is crucial in many applications such as detection of explosive devices in defense and security systems [1], [2]. This paper is concerned with the mixture estimation problem in Raman spectroscopy, where a spectrum is obtained for a given chemical using a laser to energize its molecules. Once energized, the molecules release a scattered light with a unique spectrum [3]. Specifically, a small fraction of the scattered photons have a wavelength different from that of the incoming laser light. Counting the number of photons at each wavelength provides a spectrum for a particular chemical. The spectra of different chemicals are collected in a database, called a chemical library.Traditional spectroscopy-based mixture estimation methods sample the entire spectra of a chemical mixture and use traditional estimation theory to perform the mixture estimation [4]. Another approach has been introduced recently using an adaptive spectrometer together with sequential hypothesis testing [5]. This approach provides a basis for using compressive sensing for chemical mixture estimation.

Compressive sensing aims at recovering sparse signals from a set of linear projections. In the formulation considered here, a vector having entries corresponding to the fraction of each chemical present in a mixture can be seen as a sparse signal to be recovered and the chemical library as a transformation of

L. Danjean (Corresponding author), B. Vasic and Michael W. Marcellin are with the Department of Electrical and Computer Engineering, University of Arizona, 1230 E. Speedway Boulevard, Tucson, AZ 85721, USA. E-mails: \{danjean,vasic,marcellin\}@ece.arizona.edu

D. Declercq is with ETIS, ENSEA/Univ. Cergy-Pontoise/ CNRS, 6, avenue du Ponceau, Cergy-Pontoise, 95014, France. E-mail: declercq@ensea.fr.

This work was funded by DARPA under the Knowledge Enhanced Compressive Measurements program (KECoM) through contract \#N66001-10-14079 and by the NSF under grants CCF-0830245 and CCF-0963726. the chemical mixture vector into the spectrum of the mixture. Practically, a limited number of chemicals are likely to be present among all the chemicals from the library, hence the sparseness of the mixture vector.

In this letter we propose the use of an appropriate measurement (projection) matrix for use in the adaptive spectrometer of [5] which allows the recovery of the chemical mixture vector from compressive observations of the spectrum of the mixture. A global measurement matrix is chosen to be a sparse non-negative matrix obtained from low-density parity-check (LDPC) code designs. In spectroscopy the number of potential chemicals to be detected may be large, hence the dimension of the chemical mixture vector is often large. For this reason, the usual $\ell_{1}$-norm solution using linear programming (LP) may not be suitable in practice due to its cubic complexity.

Since Donoho's original work on compressive sensing [6], numerous reconstruction algorithms have been proposed to approach LP performance with a fraction of its complexity. Such algorithms include iterative algorithms reviewed in the survey provided in [7, Chapter 8]. In this letter we use a low-complexity iterative algorithm, called the interval-passing algorithm (IPA) [8]. The IPA uses sparse measurement matrices and is well suited for the chemical mixture problem as it is designed for non-negative sparse vectors. Together with our sparse measurement matrices obtained via LDPC matrix design, the IPA allows perfect recovery of very high dimensional mixture vectors in the noise free case.

The rest of the letter is organized as follows. Section II provides preliminaries on compressive sensing, LDPC matrices, and the IPA. In Section III we provide background on spectral estimation and present the mixture estimation problem. Section IV describes the proposed compressive sensing scheme as well as the design of sparse measurement matrices. In Section V we present reconstruction results, as well as a brief discussion.

\section{Preliminaries}

\section{A. Compressive sensing}

Let $\mathbf{x} \in \mathbb{R}^{n}$ be an $n$-dimensional $k$-sparse signal, i.e., a signal with at most $k$ non-zero elements, and let $\mathbf{A} \in \mathbb{R}^{m \times n}$ be a measurement matrix. Compressive sensing [6] is concerned with the recovery of $\mathbf{x}$ from measurements $\mathbf{y}=\mathbf{A x} \in \mathbb{R}^{m}$ where $m<n$ and $k \ll n$. One approach to recover $\mathbf{x}$ from the measurements $\mathbf{y}$, is to find a signal $\mathbf{x}$ that satisfies $\mathbf{y}=\mathbf{A} \mathbf{x}$ from all the $k$-sparse signals having $\left(\begin{array}{l}n \\ k\end{array}\right)$ possible support sets which is known to be an NP-hard problem. This non-tractable problem is called $\ell_{0}$-minimization. LP technique introduced by 
Chen et al. [9], called Basis Pursuit, provides a minimum $\ell_{1}$ norm solution $\hat{\mathbf{x}}$ for $\mathbf{x}$. Provided that the measurement matrix A satisfies the Restricted Isometry Property [10], $\hat{\mathbf{x}}=\mathbf{x}$ with overwhelming probability.

\section{B. LDPC codes}

An $(n, n-m)$ binary LDPC code [11] is defined as the null space of a parity-check matrix $\mathbf{H}$ of size $m \times n$. $\mathbf{H}$ is the adjacency matrix of its equivalent bipartite graph $\mathcal{G}$, called a Tanner graph. The Tanner graph $\mathcal{G}$ of an LDPC code is defined as $\mathcal{G}=(V \cup C, E)$, where $V$ is the set of $n$ variable nodes corresponding to the $n$ columns of $\mathbf{H}$, and $C$ is the set of $m$ check nodes corresponding to the $m$ rows of $\mathbf{H}$. $E$ is the set of edges in $\mathcal{G}$, and an edge connects the variable node $v_{j}$ to the check node $c_{i}$ iff $h_{i j} \neq 0$ where $h_{i j}$ is the element at the $i^{t h}$ row and $j^{t h}$ column in $\mathbf{H}$. The code is said to be $\left(d_{v}, d_{c}\right)$ regular if all variable (resp. check) nodes in $\mathcal{G}$ have the same degree $d_{v}\left(\right.$ resp. $\left.d_{c}\right)$.

\section{Message-passing algorithms}

The first link between LDPC matrices for channel coding and LDPC matrices used as measurement matrices was established in [12] where it has been shown that good LDPC matrices for LP decoding are also good measurement matrices for LP based reconstruction in compressive sensing. These first results give a basis to provide a natural relation between measurement matrices for compressive sensing and LDPC matrices, which explains why several iterative algorithms have been recently proposed to recover sparse signals using messagepassing. The first class of message-passing algorithms, such as the approximate message-passing algorithm (AMP) presented in [13], are iterative thresholding algorithms. Other messagepassing algorithms exist in the literature such as list decoding and multiple-basis belief propagation [14], sparse matching pursuit [15], verification decoding [16] or interval-passing [8] that we present in the next section.

\section{The interval-passing algorithm}

The IPA has been analyzed in [8]. Notably, it was shown that the IPA is a good complexity/performance trade-off between LP reconstruction and simple verification decoding [16]. The IPA is an iterative algorithm. Messages are associated with the graphical representation of the measurement matrix to perform the reconstruction. Let $V=\left\{v_{1}, v_{2}, \ldots, v_{n}\right\}$ and $C=\left\{c_{1}, c_{2}, \ldots, c_{m}\right\}$ be respectively the sets of variable nodes and measurement nodes in the graphical representation of the non-negative real-valued measurement matrix $\mathbf{A}=\left\{a_{j, i}\right\}$ with $1 \leq j \leq m$ and $1 \leq i \leq n$.

In the IPA, the messages passed through edges are intervals $[\mu, M]$ corresponding to lower and upper bounds of the estimation of the connected variable node. At each iteration $l$, the message update from the variable node $v_{i}$ to the measurement node $c_{j}$ is given by:

$$
\begin{aligned}
\mu_{v_{i} \rightarrow c_{j}}^{(l)} & =\max _{c_{j}^{\prime} \in \mathcal{N}\left(v_{i}\right)}\left(\mu_{c_{j}^{\prime} \rightarrow v_{i}}^{(l-1)}\right) \times a_{j, i} \\
M_{v_{i} \rightarrow c_{j}}^{(l)} & =\min _{c_{j}^{\prime} \in \mathcal{N}\left(v_{i}\right)}\left(M_{c_{j}^{\prime} \rightarrow v_{i}}^{(l-1)}\right) \times a_{j, i}
\end{aligned}
$$

and the messages from the measurement node $c_{j}$ to the variable node $v_{i}$ are updated as:

$$
\begin{aligned}
\mu_{c_{j} \rightarrow v_{i}}^{(l)} & =\max \left(0, \frac{y_{j}-\sum_{v_{i}^{\prime} \in \mathcal{N}\left(c_{j}\right) \backslash\left\{v_{i}\right\}} M_{v_{i}^{\prime} \rightarrow c_{j}}^{(l)}}{a_{j, i}}\right) \\
M_{c_{j} \rightarrow v_{i}}^{(l)} & =\frac{y_{j}-\sum_{v_{i}^{\prime} \in \mathcal{N}\left(c_{j}\right) \backslash\left\{v_{i}\right\}} \mu_{v_{i}^{\prime} \rightarrow c_{j}}^{(l)}}{a_{j, i}}
\end{aligned}
$$

where $\mathcal{N}\left(v_{i}\right)$ (resp. $\mathcal{N}\left(c_{j}\right)$ ) is the set of measurement (resp. variable) nodes which are the neighbors of $v_{i}$ (resp. $c_{j}$ ). More details on this algorithm can be found in [8].

\section{The MiXture Estimation Problem}

Raman spectroscopy [3] relies on the uniqueness of the proportion of photons scattered inelastically from an energized molecule. A photon is said to be scattered inelastically if its wavelength is different from the wavelength of the incoming energizing laser light. Counting the number of photons with this property at each wavelength for molecules composing a chemical provides a Raman spectrum which can be used as a fingerprint for the identification of the given chemical.

Our Raman spectra estimation approach is supervised and based on the sensor of [5] which acquires compressive measurements. Unlike unsupervised approaches where spectral estimation must be accomplished from measurements without any prior information, our supervised approach assumes complete knowledge of each possible chemical spectra. Both approaches have advantages and drawbacks. In the supervised approach the prior information about the chemicals is very helpful for the reconstruction, but this knowledge needs to be acquired beforehand and the considered chemicals need to be carefully chosen in order not to disregard a chemical which can be present. In the unsupervised approach no prior work is required but the measurements process can be tedious and complex in some real time applications.

The mixture estimation problem aims to estimate the proportion of each chemical present in a mixture. As mentioned above, the spectrum of each possible chemical present is assumed. For a given chemical $\xi_{j}$, the spectrum is represented by the vector $\mathbf{S}_{j}=\left[s_{1, j}, s_{2, j}, \ldots, s_{n_{s}, j}\right]^{T}$ where $s_{i, j}$ represents the number of photons scattered inelastically for chemical $\xi_{j}$ in the $i$ th spectral channel, $i=1, \ldots, n_{s}$. Organizing these vectors as a matrix of size $n_{s} \times n_{c}$ results in a chemical library $\mathbf{L}=\left[\mathbf{S}_{1}, \mathbf{S}_{2}, \ldots, \mathbf{S}_{k}, \ldots, \mathbf{S}_{n_{s}}\right]$,

$$
\mathbf{L}=\left(\begin{array}{cccc}
s_{1,1} & s_{1,2} & \ldots & s_{1, n_{c}} \\
\vdots & \vdots & \vdots & \vdots \\
s_{n_{s}, 1} & s_{n_{s}, 2} & \ldots & s_{n_{s}, n_{c}}
\end{array}\right) .
$$

A mixture of chemicals is modeled by a vector $\mathbf{x}=$ $\left[x_{1}, \ldots, x_{n_{c}}\right]^{T}$, in which $x_{j}$ is the fraction of chemical $\xi_{j}$ present in the mixture. No chemical outside the library can be present, thus $\|\mathbf{x}\|_{1}=1$. Moreover, we assume that only $k \ll n_{c}$ chemicals are present, which implies $\|\mathbf{x}\|_{0}=k$. The resulting spectrum for the mixture of chemicals is modeled by the $n_{s} \times 1$ vector

$$
\mathbf{z}=\mathbf{L} \mathbf{x} .
$$


Although the vector $\mathrm{x}$ is sparse, the chemical library and $\mathbf{z}$ are not. Indeed a spectrum may not have a single non-zero spectral component.

From the uniqueness of the spectrum for a given chemical, we show in what follows that an appropriate choice of measurement matrix can be used to obtain compressive spectrometer measurements. The chemical mixture vector can then be recovered from these compressive measurements via IPA presented above.

\section{Application of Compressive Sensing to Raman SPECTROSCOPY}

We now present the proposed compressive sensing scheme. Instead of measuring the vector $\mathbf{z}$ directly, the compressive spectrometer of [5] measures linear combinations of the spectral channels from the mixture spectrum via a measurement matrix A of size $m \times n_{s}$. The measurement vector (output of the spectrometer) is denoted by the vector $\mathbf{y}$

$$
\mathbf{y}=\mathbf{A z}=\mathbf{A L x}
$$

of size $m \times 1$. When $m<n_{s}$, the system is compressive compared to measuring $\mathbf{z}$ directly. In the problem defined above, from the observation vector $\mathbf{y}$, we want to recover $\mathbf{x}$ directly without necessarily recovering $\mathbf{z}$ first. In other words, we formulate the problem as $\mathbf{y}=\mathbf{M x}$, with $\mathbf{M}=\mathbf{A L}$. Given that the chemical library $\mathbf{L}$ is fixed and known, by choosing the elements of the matrix A properly, we can make $\mathbf{M}$ a nonnegative sparse matrix which is a necessary condition for use of the IPA for reconstruction. The matrix A then governs the way that measurements (of the mixture spectrum) are taken by the spectrometer, while $\mathbf{M}$ governs the recovery of $\mathbf{x}$. If $m>n_{c}$, Eq. 6 is typically over-determined with respect to $\mathrm{x}$, and least square techniques are easily employed. The more interesting case is when $m<n_{c}$ and the system is undetermined (compressive) with respect to $\mathbf{x}$. This is the case treated in what follows.

\section{A. Design of the matrix $\mathbf{A}$}

As mentioned previously, the IPA has very low computational complexity, but requires that the measurement matrix be sparse. With respect to the mixture vector $\mathbf{x}$, the measurement matrix is $\mathbf{M}=\mathbf{A L}$. The design of a matrix $\mathbf{A}$ to obtain a sparse matrix $\mathbf{M}$ is not a trivial problem. We propose instead to make a choice for $\mathbf{M}$, and then design $\mathbf{A}$ such that the product $\mathbf{A L}$ produces the chosen sparse matrix $\mathbf{M}$. The main idea is to ignore all but $n_{c}$ spectral channels. The $n_{c}$ rows in $\mathbf{L}$ corresponding to the $n_{c}$ selected channels then form a square matrix (denoted $\mathbf{L}_{\mathbf{s}}$ ). If the columns of $\mathbf{L}$ are independent (which will be the case in our example), the rank of the matrix $\mathbf{L}_{\mathbf{s}}$ is $n_{c}$, meaning that the matrix $\mathbf{L}_{\mathbf{s}}$ is invertible. We then let

$$
\mathbf{M L}_{\mathbf{s}}^{-1}=\mathbf{A}_{\mathbf{s}}=\left[\begin{array}{lllll}
\mathbf{A}_{\varphi_{1}} & \ldots & \mathbf{A}_{\varphi_{j}} & \ldots & \mathbf{A}_{\varphi_{n_{c}}}
\end{array}\right]
$$

and define $\mathbf{A}$ from the columns of $\mathbf{A}_{\mathbf{s}}$ as:

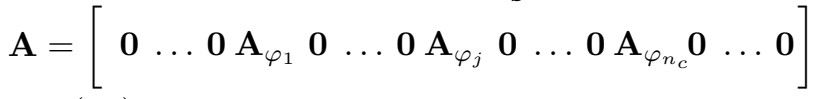

where $\left(\varphi_{j}\right) j=1, \ldots, n_{c}$ is the sequence of spectral channels which are not disregarded. It is straightforward to verify that

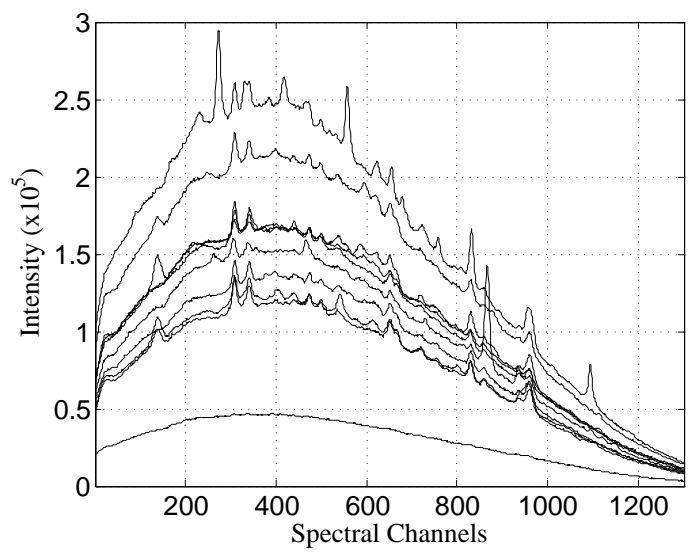

Fig. 1. Raman spectra of 10 pharmaceutical chemicals.

$\mathbf{A L}=\mathbf{A}_{\mathbf{s}} \mathbf{L}_{\mathbf{s}}=\mathbf{M L}_{\mathbf{s}}^{-1} \mathbf{L}_{\mathbf{s}}=\mathbf{M}$ as desired. The sequence of $\varphi_{j}$ s can be chosen randomly or in a predetermined fashion. Due to page limits we omit the details regarding this choice, however experiments have shown that this choice does not involve any significant loss/gain in the performance of the reconstruction.

In the next subsection we discuss briefly the IPA reconstruction performance, providing insight on the properties that $\mathbf{M}$ should satisfy to obtain the best achievable performance.

\section{B. Design of the matrix $\mathrm{M}$}

In order to provide a brief analysis of the theoretical reconstruction possibilities, we recall that, from the IPA standpoint, a chemical mixture is a $k$-sparse non negative signal $\mathbf{x}$ observed through a sparse measurement matrix M. The IPA is used to reconstruct $\mathbf{x}$. However we know that if the graphical representation of $\mathbf{M}$ contains some specific topologies (small size stopping sets), the IPA will fail in some cases [8].

Definition 1 ([17]). A stopping set (SS) $T$ is a subset of the set of variable nodes $V$ of $\mathbf{M}$ such that all neighbors of $T$ are connected to $T$ at least twice. The cardinality of a SS is called the size of the SS.

Theorem 1. Let $\mathbf{M} \in \mathbb{R}^{m \times n}$ be a sparse measurement matrix and let $k$ be the size of a SS in $\mathbf{M}$ with support $T=\left\{i_{1}, i_{2}, \ldots, i_{k}\right\}$, where $1 \leq i_{l} \leq n_{c}, l=1,2, \ldots, k$. Let $\mathbf{x}=\left[x_{1}, x_{2}, \ldots, x_{n_{c}}\right]^{T} \in \mathbb{R}^{n_{c}}$ be a chemical mixture vector. If the support of the vector $\mathbf{x}$ is exactly the support $T$ of a SS in $\mathrm{M}$, then, the IPA cannot recover the chemical mixture.

Proof: The proof is identical to [8, Theorem 1].

This theorem provides limitations on the reconstruction of chemical mixtures using the IPA, and a link with the analysis of the failures of LDPC codes under iterative decoding on the binary erasure channel. The knowledge of SSs in the measurement matrix $\mathbf{M}$ gives an upper bound to the minimum number of SSs. More work from [8] can be directly applied, especially regarding the conditions for a correct reconstruction is the support of the vector $\mathbf{x}$ is included in a SS.

\section{Simulation Results AND Discussion}

We present here simulation results for the mixture estimation problem using the IPA for a chemical library from real 


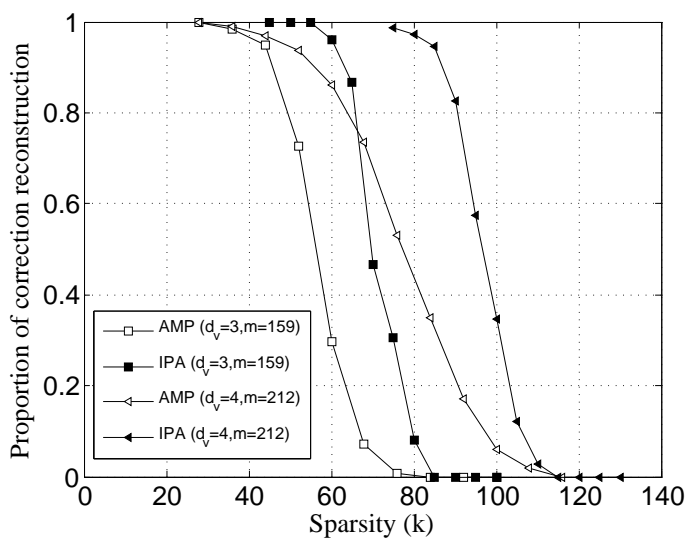

Fig. 2. Proportion of correct reconstructions versus number of chemicals present $(k)$ using different sparse measurement matrices $\mathbf{M}$.

measurements of different pharmaceutical chemicals. Fig. 1 presents an example of 10 spectra from the chemical library. The library is composed of $n_{c}=265$ different chemicals each having $n_{s}=1300$ spectral samples. The size of the chemical library $\mathbf{L}$ is then $1300 \times 265$. Note that in this example $n_{s}>n_{c}$, but no restrictions exist on $n_{s}$ nor $n_{c}$ to set our compressive scheme as long as $m<n_{c}$. Each Raman spectra in the library is unique, although they may be very similar. Indeed for many chemicals, on a range of spectral channels, the behavior is similar (peaks of plateau around same channels). The sequence of $\varphi_{j}$ s has been selected here by taking the first $n_{c}$ peaks common to the majority of chemicals.

As in [8], the measurement matrices $\mathbf{M}$ used in this letter are designed from the code construction technique of structured LDPC matrices of [18]. Each designed matrix $\mathbf{M}$ is then a block matrix of circularly shifted versions of the identity matrix of size $p \times p$. We used this code construction technique as it is very simple to use, but other code construction techniques can be used and give similar results. As an example, let $p=53$ and let:

$$
\mathbf{M}=\left(\begin{array}{ccccc}
\mathbf{I}_{\mathbf{0}} & \mathbf{I}_{0} & \mathbf{I}_{0} & \mathbf{I}_{0} & \mathbf{I}_{\mathbf{0}} \\
\mathbf{I}_{\mathbf{0}} & \mathbf{I}_{14} & \mathbf{I}_{1} & \mathbf{I}_{36} & \mathbf{I}_{37} \\
\mathbf{I}_{0} & \mathbf{I}_{3} & \mathbf{I}_{\mathbf{4}} & \mathbf{I}_{38} & \mathbf{I}_{\mathbf{4 2}}
\end{array}\right)
$$

where $\mathbf{I}_{j}$ denotes the identity matrix circularly shifted by a factor $j$. This choice yields a $(3,5)$-regular LDPC matrix ${ }^{1}$. Indeed, there are exactly $d_{v}=3$ " 1 "s per column (each column block is composed of 3 shifted versions of the identity matrix), and $d_{c}=5$ " 1 "s per row (each row block is composed of 5 shifted versions of the identity matrix). Finally, $\mathbf{M}$ is of size $m \times n_{c}=p d_{v} \times p d_{c}=m \times n_{c}=159 \times 265$. The value of $p=53$ in the example is a prime factor of $n_{c}=265$.

Fig. 2 presents simulation results showing the proportion of correct reconstructions between the original chemical mixtures and the reconstructed ones in a noise free environment. For each sparsity $k$, random chemical mixtures (or equivalently random $k$-sparse vectors $\mathbf{x}$ with $\|\mathbf{x}\|_{1}=1$ ) are generated. The generation of a vector $\mathbf{x}$ is done by first creating a non-negative unit $\ell_{1}$-norm vector of length $k$ and then assign randomly each of the $k$ generated values among the $n$ values of $\mathbf{x}$, the

\footnotetext{
${ }^{1}$ We use binary measurements matrices for the sake of simplicity as the use of non-negative values give the same results [8]
}

$n-k$ other values of $\mathrm{x}$ being set to 0 . The measurements $\mathbf{y}$ are then obtained via $\mathbf{y}=\mathbf{M x}$. A maximum of 50 IPA iterations are performed. A mixture is said to be correctly recovered if each $x_{j} j=1, \ldots, n_{c}$ is recovered with absolute error no greater than $10^{-6}$. Fig. 2 demonstrates that chemical mixtures with a large number of chemicals can be recovered exactly. Specifically, the recovery of mixtures of up to 55 chemicals can be obtained with $m=159$ measurements from the spectrometer, which represents a compression ratio of $1300: 159 \gtrsim 8: 1$. For $m=212$, it is about 75 chemicals that can be recovered, which represents a compression ratio of $1300: 212 \gtrsim 6: 1$.

We also compare the results of the IPA with the AMP. Instead of using a measurement matrix $\mathbf{M}$ which is sparse, the AMP requires $\mathbf{M}$ to be a random matrix whose elements are i.i.d. drawn from some distribution, taken here to be Gaussian. We can see that the performance is better for the IPA for both choices of measurement matrix sizes (compression ratios). The AMP does not allow, in general, for perfect recovery, hence for the AMP, we relax our criterion and say that a mixture is said to be correctly recovered if each $x_{j} j=1, \ldots, n_{c}$ is recovered with absolute error no greater than $10^{-3}$. The results for an absolute value no greater than $10^{-6}$ are poor.

The noisy case will be addressed in future publications, and first results show that with a modification of the IPA, good mean-square error reconstruction results can be obtained.

\section{ACKNOWLEDGMENTS}

The authors thank Dr. M. E. Gehm for providing the spectral library and V. Ravanmehr for her work on the IPA.

\section{REFERENCES}

[1] H.-B. Liu, H. Zhong, N. Karpowicz, Y. Chen, and X.-C. Zhang, "Terahertz spectroscopy and imaging for defense and security applications," Proc. of the IEEE, vol. 95, no. 8, pp. 1514-1527, Aug. 2007.

[2] N. Butt, M. Nilsson, A. Jakobsson, M. Nordberg, A. Pettersson, S. Wallin, and H. O andstmark, "Classification of Raman Spectra to Detect Hidden Explosives," IEEE Geosci. and Remote Sensing Lett. vol. 8, no. 3, pp. 517-521, May 2011.

[3] D. J. Gardiner, Practical Raman Spectroscopy, P. R. Graves, Ed. Springer-Verlag, Apr. 1989.

[4] R. Kopelman and W. Tan, "Near-field optical microscopy, spectroscopy, and chemical sensors," Appl. Spectroscopy Reviews, vol. 29, no. 1, pp. 39-66, Aug. 1994.

[5] D. V. Dinakarababu, D. R. Golish, and M. E. Gehm, "Adaptive feature specific spectroscopy for rapid chemical identification," Opt. Express, vol. 19, no. 5, pp. 4595-4610, Feb. 2011.

[6] D. Donoho, "Compressed sensing," IEEE Trans. Inf. Theory, vol. 52, no. 4, pp. 1289-1306, Apr. 2006.

[7] Y. C. Eldar and G. Kutyniok, Compressed Sensing: Theory and Applications. Cambridge University Press, 2012.

[8] V. Ravanmehr, L. Danjean, B. Vasic, and D. Declercq, "Interval-passing algorithm for non-negative measurement matrices: Performance and reconstruction analysis," IEEE J. Emerging and Sel. Topics in Circuits and Syst., vol. 2, no. 3, pp. 424-432, Oct. 2012.

[9] S. S. Chen, D. L. Donoho, and M. A. Saunders, "Atomic decomposition by basis pursuit," SIAM Journal on Scientific Computing, vol. 20, no. 1, pp. 33-61, Aug. 1998.

[10] E. J. Candes and T. Tao, "Decoding by linear programming," IEEE Trans. Inf. Theory, vol. 51, no. 12, pp. 4203-4215, Dec. 2005.

[11] R. G. Gallager, Low Density Parity Check Codes. Cambridge, MA, USA: M.I.T. Press, 1963.

[12] A. G. Dimakis, R. Smarandache, and P. O. Vontobel, "LDPC codes for compressed sensing," IEEE Trans. Inf. Theory, no. 5, pp. 3093-3114, May. 2012. 
[13] D. L. Donoho, A. Maleki, and A. Montanari, "Message passing algorithms for compressed sensing," Proc. Nat. Academy of Sciences, vol. 106, no. 45, pp. 18 914-18 919, Sep. 2009.

[14] H. V. Pham, W. Dai, and O. Milenkovic, "Sublinear compressive sensing reconstruction via belief propagation decoding," in Proc. IEEE Int. Symp. Inform. Theory, Seoul, Korea, Jun. 28-Jul. 3 2009, pp. 674-678.

[15] R. Berinde, P. Indyk, and M. Ruzic, "Practical near-optimal sparse recovery in the L1 norm," in Proc. Allerton Conf. on Commun., Control, and Computing, Urbana-Champaign, IL, USA, Sep. 2008, pp. 198-205.

[16] F. Zhang and H. D. Pfister, "Verification decoding of high-rate LDPC codes with applications in compressed sensing," IEEE Trans. Inf. Theory, vol. 58, no. 8, pp. 5042-5058, Aug. 2012.

[17] C. Di, D. Proietti, I. Telatar, T. Richardson, and R. Urbanke, "Finitelength analysis of low-density parity-check codes on the binary erasure channel," IEEE Trans. Inf. Theory, vol. 48.

[18] D. V. Nguyen, S. K. Chilappagari, B. Vasic, and M. W. Marcellin, "On the construction of structured LDPC codes free of small trapping sets," IEEE Trans. Inf. Theory, vol. 58, Apr. 2012. 